\title{
AN IN-VITRO STEREOMICROSCOPIC COMPARISON OF PROTAPER RETREATMENT, MTWO RETREATMENT AND HEDSTROM FILES IN REMOVAL OF AN EPOXY RESIN-BASED SEALER AND MTA- BASED SALICYLATE RESIN SEALER DURING ENDODONTIC RETREATMENT
}

\author{
N. M. Dhanyakumar1, Vasundhara Shivanna ${ }^{2}$, Sushant Sharma ${ }^{3}$
}

${ }_{1}^{1}$ Professor and Dean of Examination, Department of Conservative Dentistry and Endodontics, College of Dental Sciences, Davangere, Karnataka, India.

2Professor and HOD, Department of Conservative Dentistry and Endodontics, College of Dental Sciences, Davangere, Karnataka, India. 3 Postgraduate Student, Department of Conservative Dentistry and Endodontics, College of Dental Sciences, Davangere, Karnataka, India.

\section{BACKGROUND}

\section{ABSTRACT}

Success of non-surgical endodontic retreatment is dependent on the effective removal of root canal filling material. Not many studies have compared the effectiveness of different file systems in removing root canal filling when obturated with different resinbased sealers.

The aim of the study was to compare the efficacy of Protaper retreatment (PTR) files, Mtwo retreatment (MtwoR) files and Hedstrom $(\mathrm{H})$ files in removal of gutta-percha and two types of resin-based sealers during endodontic retreatment.

\section{MATERIALS AND METHODS}

A randomised controlled trial was conducted on 90 extracted single rooted single canal human mandibular first premolars. All specimens were divided into 3 groups depending on the initial root canal preparation: Group I used hand K files, Group II used rotary Mtwo files and Group III used rotary Protaper Universal files. These 3 groups were further subdivided into 2 subgroups A and B: obturated with MTA Fillapex and AH plus sealer respectively. Each group and its subgroups were then subjected to retreatment. The remnant root filling material in each group and subgroup was measured by using stereomicroscope. MannWhitney and Kruskal-Wallis test were used for data analysis.

\section{RESULTS}

PTR files were the most effective in endodontic retreatment showing significant difference as compared to MtwoR and Hand $\mathrm{H}$ files. Root canals obturated with MTA Fillapex sealer showed significantly less root filling remnants.

\section{CONCLUSION}

PTR files were more efficient in removing root canal filling material as compared to MtwoR files and Hand H files. MTA Fillapex sealer left lesser remnants than AH plus subgroup.

\section{KEY WORDS}

Protaper Retreatment Files; Mtwo Retreatment Files; Hedstrom Files; MTA Fillapex; AH Plus; Retreatment.

HOW TO CITE THIS ARTICLE: Dhanyakumar NM, Shivanna V, Sharma S. An in-vitro stereomicroscopic comparison of protaper retreatment, Mtwo retreatment and Hedstrom files in removal of an epoxy resin-based sealer and MTA-based salicylate resin sealer during endodontic retreatment. J. Evolution Med. Dent. Sci. 2018;7(42):4526-4530, DOI: 10.14260/jemds/2018/1010

\section{BACKGROUND}

Failure of the root canal treatment is caused due to insufficient cleaning, shaping and obturation or loss of coronal seal causing re-infection in the root canals. Irrespective of the aetiology, the result is leakage and contamination by bacteria.[1] Complex canal anatomies and persistence of microorganisms describe $8 \%$ of treated canals undergoing failure.[2]

Gutta-percha in conjunction with sealer is the obturating material used most commonly. ${ }^{[3]}$ Non-surgical retreatment is a procedure in which the gutta-percha and sealer is removed, so that a thorough cleaning, shaping and re-obturation of the

'Financial or Other Competing Interest': None.

Submission 31-08-2018, Peer Review 26-09-2018,

Acceptance 03-10-2018, Published 15-10-2018.

Corresponding Author:

Dr. Sushant Sharma,

Room No. 9B,

Department of Conservative Dentistry and Endodontics,

Davangere-577004, Karnataka, India.

E-mail: sushant13jan@gmail.com

DOI: $10.14260 /$ jemds $/ 2018 / 1010$

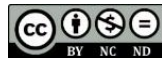

root canals can be performed. The goal of this procedure is to restore the periapical tissues to healthy condition, which demands a thorough root canal filling removal reaching the working length again, chemo-mechanical disinfection and reobturation of root canals.[4] $65 \%$ to greater than $80 \%$ success rate has been seen with non-surgical retreatment.[3]

Protaper Retreatment file system (PTR) (Dentsply Maillefer) has D1, D2 and D3 files. D1 has size 30, .09 taper with length of $16 \mathrm{~mm}$, D2 has size 25, .08 taper with $18 \mathrm{~mm}$ length and D3 has size 20, .07 taper with $22 \mathrm{~mm}$ length. Root filling material is removed from the cervical third by D1, middle third by D2 and apical third by D3 files. ${ }^{[5]}$

Mtwo retreatment files (MtwoR) (VDW) consists of R1 and R2. R1 has size 15, .05 taper and R2 has size 25, .05 taper, both having $21 \mathrm{~mm}$ length. They have S-shaped cross-section, two cutting edges and short pitch length for efficient cutting.[6]

AH plus comprises of an epoxide and amine paste-paste system being conveyed in two tubes. Bismuth Radiopaque fillers and Aerosil form the components of the epoxide paste, while three different kinds of amines, radiopaque fillers and aerosil form the components of amine paste. $\mathrm{AH}$ plus is 
marked by high mechanical properties, radiopacity, long shelf life and low polymerisation shrinkage and solubility.[7]

MTA Fillapex comprises of salicylate resin, diluting resin, natural resin, bismuth trioxide, nanoparticulated silica and MTA along with pigments.[8] The manufacturer states it to have extremely good radiopacity, convenient handling, long working time, less solubility, excellent sealing through expansion on setting.[9]

Till date, no study has been performed which has compared the efficacy of PTR files, MtwoR files and H files in removal of AH plus and MTA Fillapex sealer along with guttapercha during endodontic retreatment.

Thus, the aim of this study was to compare the efficacy of PTR files, MtwoR files and $\mathrm{H}$ files in the removal of AH plus and MTA Fillapex sealer along with gutta-percha during endodontic retreatment.

\section{MATERIALS AND METHODS \\ Study Design}

A randomised controlled trial.

\section{Selection of Teeth}

90 single rooted extracted human mandibular first premolars with single, straight and patent canals were collected, stored in normal saline at room temperature.

\section{Exclusion Criteria}

Teeth with cracks, resorption, dilacerations, multiple canals, calcifications, immature apices and developmental disturbances were excluded.

\section{Disinfection of Specimens}

All teeth were cleaned with ultrasonic scaler accompanied by immersion in $5.25 \%$ sodium hypochlorite for 15 minutes.

\section{Methodology}

All the specimens were decoronated using diamond disc at the level of cementoenamel junction for standardisation of all samples to $15 \mathrm{~mm}$ length. Patency was established with $15 \mathrm{~K}$ file till the file was just visible at the apex. Working length was determined by deducting $1 \mathrm{~mm}$ from this length.

All the teeth were divided into 3 groups of 30 teeth each depending on the biomechanical preparation of the specimens.

- Group I: Root canals were prepared with hand K files using step-back technique upto size $30 \mathrm{~K}$ till the working length.

- Group II: Root canals were prepared with Mtwo rotary files using crown-down technique in the sequence of size $15, .05$ taper, size 20, .06 taper, size 25, .06 taper followed by size $30, .05$ taper according to manufacturer's recommendations till the working length.

- Group III: Root canals were prepared with Protaper Universal rotary files using crown-down technique in sequence of S1, Sx, F1, F2 and F3 according to manufacturer's recommendations till the working length.

Irrigation was done using $5 \mathrm{~mL}$ of $5.25 \% \mathrm{NaOCl}$ being used in between instruments. Saline was used as the final irrigant. Root canals of all the specimens were dried with paper points. Each group was further subdivided into 2 subgroups of 15 teeth each on the basis of sealer used for obturation-

- Subgroup A: Teeth obturated with MTA Fillapex and gutta-percha using single cone technique.

- Subgroup B: Teeth obturated with AH plus and guttapercha using single cone technique.

Root canal orifices of all the specimens were sealed with glass ionomer cement followed by storage of all teeth at $37^{\circ} \mathrm{C}$ and $100 \%$ humidity for a week in an incubator to allow complete setting of the sealers. Glass ionomer cement was removed from the access cavities before the retreatment procedure.

\section{Retreatment}

Subgroups IA and IB: Retreatment of root canals was done using hand Hedstrom files in the sequence of size 30, 25 and 20 in a circumferential motion.

Subgroups IIA and IIB: Retreatment of root canals was done using Mtwo Retreatment files in the sequence of R1, R2 at $280 \mathrm{rpm}$ and $1.2 \mathrm{Ncm}$ torque using simultaneous technique.

Subgroups IIIA and IIIB: Retreatment of root canals was done using Protaper Retreatment files in the sequence of D1, D2, D3 at $500 \mathrm{rpm}$ and $2 \mathrm{Ncm}$ torque.

Normal saline was used to do the irrigation in between the instruments. Retreatment was considered to be complete when no gutta-percha was seen on the flutes of the file and when the working length was reached. For each tooth, new set of instruments were used.

\section{Sample Evaluation}

The teeth were grooved longitudinally on the buccal and lingual surfaces of each specimen with a diamond disc and a chisel was used to split them into two halves. Each root half was observed under stereomicroscope at $8 \mathrm{X}$ magnification and photographed. A grading system suggested by Somma et al was used to score the remaining root canal filling material in the coronal, middle and apical third of each root half.

Score 0: $0-25 \%$ of the remaining root canal filling material covering the surface

Score 1: $25-50 \%$ of the remaining root canal filling material covering the surface

Score 2: $50-75 \%$ of the remaining root canal filling material covering the surface

Score 3: $75-100 \%$ of remaining root canal filling material covering the surface.

No effort was made to distinguish between gutta-percha and sealer.

\section{Statistical Analysis}

Statistical analysis of data was done using Mann-Whitney test and Kruskal-Wallis test. Result was statistically significant when the p-value $<0.05$.

\section{RESULTS}

Comparison between the mean amount of remaining root canal filling material among three groups and their 
comparison on the basis of the sealer used for obturation is shown in Table 1 and 2 respectively. Irrespective of the sealer used, there was significant difference among the three groups with PTR files showing the least amount of residual filling material followed by MtwoR and then the $\mathrm{H}$ files. Teeth obturated with MTA Fillapex sealer showed significantly less residual root filling material. In the apical one-third, MtwoR fared better than PTR and $\mathrm{H}$ files. None of the groups could result in complete removal of the root canal filling.

\begin{tabular}{|c|c|c|c|c|c|c|}
\hline Groups & Subgroups & N & Median & IQR & Z & P-value \\
\hline I & A & 15 & 2 & 1 & 2.297 & $0.029^{*}$ \\
\hline & B & 15 & 3 & 1 & & \\
\hline II & A & 15 & 1 & 0 & 3.162 & $0.007^{*}$ \\
\hline & B & 15 & 2 & 1 & & \\
\hline III & A & 15 & 0 & 1 & 3.529 & $0.001^{*}$ \\
\hline & B & 15 & 1 & 0 & & \\
\hline
\end{tabular}

Table 1. Comparison between Mean Amount of remaining Root Filling Material among Three Groups

*Significant difference at value $<0.05$.

\begin{tabular}{|c|c|c|c|c|}
\hline Groups & N & Median & IQR & P-value \\
\hline IA & 15 & 2 & 1 & \\
\hline IIA & 15 & 1 & 0 & $0.000^{*}$ \\
\hline IIIA & 15 & 0 & 1 & \\
\hline IB & 15 & 3 & 1 & \\
\hline IIB & 15 & 2 & 1 & $0.001^{*}$ \\
\hline IIIB & 15 & 1 & 0 & \\
\hline
\end{tabular}

\section{Table 2. Comparison between Mean Amount of remaining} Root Canal Filling Material among Three Groups on the basis of Sealer used for Obturation

*Significant difference at value $<0.05$.

\section{DISCUSSION}

Endodontic treatment has a success rate of 86 - 98\%. Persistent bacteria, under and overextended root canal fillings, inadequate coronal seal, missed canals and iatrogenic errors caused during instrumentation are causes of endodontic failure.[10] The main purpose of endodontic retreatment is to halt the infectious process by removing the root canal filling material, enabling chemomechanical reinstrumentation and disinfection. ${ }^{[11]}$

All the specimens were decoronated to do the standardisation by removing factors like tooth crown anatomy and root canal's access.[6]

Single cone technique was used in this study because single cone in combination with endodontic cement forms a uniform mass, is simpler, shortens time for obturation as no accessory cones are required, reduces pressure applied to root canal walls and reduces operator fatigue.[12] $\mathrm{AH}$ plus sealer was used, because it is a gold standard with which all new sealers are compared and is popular for its good sealing ability, short setting time and good adhesive property. MTA Fillapex sealer was used because it forms apatite like crystalline deposits, has high flow rate, low film thickness, is not affected adversely by heat, has antibacterial properties and has a tendency to maintain relative constant calcium release for 14 days.[13]

Different techniques that have been advocated for retreatment are hand instruments alone or in combination with heat or chemicals, rotary instruments and ultrasonic instruments. ${ }^{[1]}$ Nickel Titanium rotary files have been used because of their safety, efficacy and cleaning ability.
Moreover, they take less time to remove gutta-percha than hand instruments.[14] Hedstrom files have been used for retreatment, because of its ability to remove gutta-percha in larger pieces.[15]

PTR has showed the maximum amount of gutta-percha and sealer removal because of its specific convex flute design, D1 having a cutting tip which causes effective penetration into the root filling material in the coronal third, its tendency to pull the root filling material into its flutes and push it coronally towards orifice. ${ }^{[14]}$ Moreover D1, D2, D3 owing to their variable tip diameter and progressive taper touch, only specific part of the root canal and they not only cut guttapercha but also the superficial layer of dentin.[16] PTR has shown a better result than Mtwo $\mathrm{R}$ because of its greater internal mass and greater taper which results in larger root canal area being touched and prepared.[2]

MtwoR files have a positive rake angle and two cutting edges. MtwoR files showing more amount of root filling remnant is consistent with the previous study, as it uses single length preparation technique in spite of crown-down technique which leads to greater root canal filling remnants during endodontic retreatment.[16]

PTR and MtwoR files showed better results than Hand $\mathrm{H}$ files, because the heat generated during rotary motion causes plasticisation of the gutta-percha making the gutta-percha removal very convenient.[14] Hand $H$ files leaving the maximum root filling remnant is consistent with the result of another study as they are stiffer than rotary instruments, so cannot be efficiently directed towards the walls of the root canal leading to more amount of root filling material remnants. ${ }^{[4]}$

PTR files were more effective than MtwoR files in the coronal and the middle third of the root canal. In the coronal third, removal of root filling depends on the dental anatomy and the speed of rotation of the rotary file. PTR rotates at a greater speed than MtwoR leading to greater amount of root filling being removed.[17] In contrast to this, MtwoR files were more effective than PTR files in the apical third. This may be attributed to the fact that in the MtwoR group, the master apical file had the size $30, .05$ taper and the final retreatment file used had size 25, .05 taper while in the PTR group, the master apical file had size 30, .09 taper while the final retreatment file had size of only $20, .07$ taper.

MTA Fillapex subgroup showed cleaner root canal walls after retreatment as compared to the $\mathrm{AH}$ plus subgroup. Removability of the sealers depends upon their adhesion to dentin and gutta-percha, penetration into the dentinal tubules, film thickness, dimensional changes and solubility.[17] Though MTA Fillapex has comparable dimensional stability, but has a higher solubility and film thickness than $\mathrm{AH}$ plus.[18] MTA Fillapex has low bond strength to dentin and questionable biomineralisation. Lesser remnants of root filling material in the MTA Fillapex subgroup is consistent with the result of the previous study, as when MTA contacts with the phosphate-containing fluid leads to the formation of calcium-deficient B-type carbonated apatites. These apatites formed get deposited on the collagen fibrils to form tag-like interfacial layer between MTA and dentin, which has low bond strength.[19]

The method of assessment is an important parameter in the study. Various methods like longitudinal cleavage of teeth, association of longitudinal and transverse cleavage for 
evaluation in different root thirds separately and radiographic examination have been used. Radiographic images are not competent enough to detect the fine layers of root canal filling. Moreover, it is a two-dimensional representation of three dimensional structures and may lead to magnification and distortion.[20] Clearing technique with digitised images has also been employed in some studies, but it was not used because it is time consuming and various chemicals used in it interfere with the final results. ${ }^{[14]}$ In this study, direct visual scoring of the stereomicroscopic images of longitudinal sectioned teeth had been used because it is simple and efficient.[17]

No solvent had been used during the retreatment, because the solvent softens the root filling material which enters into the irregularities of the root canal walls and the dentinal tubules which cannot be removed.[20] Solvent increases the time for root filling removal.[21]

\section{Limitations}

1. The results of this in-vitro study cannot be applied to the curved root canals, because the study was conducted in the straight root canals.

2. The bond strength of the AH plus and MTA Fillapex sealer depends on the moisture content of the dentin which is different in in-vivo conditions which subsequently affects the remnant root filling material clinically.

3. There is no standardisation of the taper of master apical file in the root canal preparation.

\section{CONCLUSION}

1. PTR files were the most effective files when comparing the total root filling material remnant because of its convex triangular flute design, each of its files ability to shape only specific area of root canal, greater internal mass and taper.

2. Root canals obturated with MTA Fillapex sealer showed the least amount of root filling material owing to its lower bond strength, greater solubility and film thickness as compared to AH plus sealer.

3. None of the instrument group could completely remove the gutta-percha and sealer from the root canal.

\section{ACKNOWLEDGEMENT}

We would like to thank the Department of Oral Pathology, College of Dental Sciences, Davanagere for providing their assistance.

\section{REFERENCES}

[1] Ruddle CJ. Nonsurgical endodontic retreatment. J Calif Dent Assoc 2004;32(6):474-84.

[2] da Silva BM, Filho FB, Leonardi DP, et al. Effectiveness of Pro Taper, D-Race, and Mtwo retreatment files with and without supplementary instruments in the removal of root canal filling material. Int Endod J 2012;45(10):927-32.

[3] Hulsmann M, Bluhm V. Efficacy, cleaning ability and safety of different rotary NiTi instruments in root canal retreatment. Int Endod J 2004;37(7):468-76.
[4] Kasam S, Mariswamy A.B. Efficacy of different methods for removing root canal filling material in retreatment- an in-vitro study. J Clin Diagn Res 2016;10(6):ZC06-10.

[5] Fariniuk LF, Azevedo MAD, Carneiro E, et al. Efficacy of protaper instruments during endodontic retreatment. Indian J Dent Res 2017;28(4):400-5.

[6] Kanaparthy A, Kanaparthy R. The comparative efficacy of different files in the removal of different sealers in simulated root canal retreatment- an in-vitro study. J Clin Diagn Res 2016;10(5):ZC130-3.

[7] Geetha RV, Veeraraghavan VP. Evaluation of antibacterial activity of five root canal sealants against enterococcus faecalis-an in vitro study. Int J Pharm Sci. Rev Res 2016;40(2):221-3.

[8] Demiriz L, Kocak MM, Kocak S, et al. Evaluation of the dentinal wall adaptation ability of MTA Fillapex using stereo electron microscope. J Conserv Dent 2016;19(3):220-4.

[9] Silva EJ, Rosa TP, Herrera DR, et al. Evaluation of cytotoxicity and physicochemical properties of calcium silicate-based endodontic sealer MTA fillapex. J Endod 2013;39(2):274-7.

[10] Tabassum S, Khan FR. Failure of endodontic treatment: the usual suspects. Eur J Dent 2016;10(1):144-7.

[11] Karamifar K, Mehrasa N, Pardis P, et al. Cleanliness of canal walls following gutta-percha removal with hand files, RaCe and RaCe plus XP-Endo finisher instruments: a photographic in vitro analysis. Iran Endod J 2017;12(2):242-7.

[12] Pereira AC, Nishiyama CK, Pinto LC. Single-cone obturation technique: a literature review. RSBO. 2012;9(4):442-7.

[13] Gad RA, Farag AM, El-Hediny HA, et al. Sealing ability and obturation quality of root canals filled with guttapercha and two different sealers. Tanta Dent J 2016;13(4):165-70.

[14] Chandrasekar, Ebenezar AV, Kumar M, et al. A comparative evaluation of gutta percha removal and extrusion of apical debris by rotary and hand files. J Clin Diagn Res 2014;8(11):ZC110-4.

[15] Imura N, Kato AS, Hata GI, et al. Comparison of the relative efficacies of four hand and rotary instrumentation techniques during endodontic retreatment. Int Endod J 2000;33(4):361-6.

[16] Lalit P, Godara N, Varma KR. Evaluation of efficiency of three NiTi instruments in removing gutta-percha from root canal during retreatment-an in vitro study. Endodontology P. 80-8.

[17] Uzunoglu E, Yilmaz Z, Sungur DD, et al. Retreatability of root canals obturated using gutta-percha with bioceramic, MTA and resin-based sealers. Iran Endod J 2015;10(2):93-8.

[18] Zhou HM, Shen Y, Zheng W, et al. Physical properties of 5 root canal sealers. J Endod 2013;39(10):1281-6.

[19] Neelakantan P, Grotra D, Sharma S. Retreatability of 2 mineral trioxide aggregate-based root canal sealers: a cone-beam computed tomography analysis. J Endod 2013;39(7):893-6. 
[20] Yadav P, Bharath MJ, Sahadev CK, et al. An in vitro CT comparison of gutta-percha removal with two rotary systems and hedstrom files. Iran Endod J 2013;8(2):59-64.
[21] Kfir A, Tsesis I, Yakirevich E, et al. The efficacy of five techniques for removing root filling material: microscopic versus radiographic evaluation. Int Endod J 2012;45(1):35-41. 